\title{
Megacyllene Casey (Coleoptera, Cerambycidae): novas sinonímias e descrição de uma nova espécie
}

\author{
Miguel A. Monné ${ }^{1,3} \&$ Dilma Solange Napp ${ }^{2,3}$
}

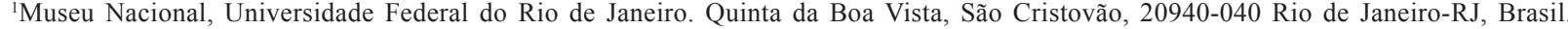
${ }^{2}$ Departamento de Zoologia, Universidade Federal do Paraná. Caixa Postal 19020, 81531-980 Curitiba-PR, Brasil.

${ }^{3}$ Pesquisador do CNPq.
\end{abstract}

\begin{abstract}
Megacyllene Casey (Coleoptera. Cerambycidae): new synonyms and description of a new species. Megacyllene ebenina sp. nov. is described from Brazil (Santa Catarina). New synonyms: M. castroi (Prosen, 1947), comb. nov., stat. nov. $=$ M. (M.) cryptofrasciata Di Iorio, 1998 syn. nov.; M. trifasciata Viana, $1994=$ M. (Sierracyllene) tafivallensis Di Iorio, 1998 syn. nov.
\end{abstract}

KeYwords. Cerambycinae; Clytini; Megacyllene; new species; new synonyms.

Resumo. Megacyllene ebenina sp. nov. é descrita do Brasil (Santa Catarina). Novos sinônimos propostos: M. castroi (Prosen, 1947), comb. nov., stat. nov. = M. (M.) cryptofrasciata Di Iorio, 1998 syn. nov.; M. trifasciata Viana, $1994=$ M. (Sierracyllene) tafivallensis Di Iorio, 1998 syn. nov.

Palavras-Chave. Cerambycinae; Clytini; espécie nova; Megacyllene; novas sinonímias.

Megacyllene Casey, 1912 é um dos maiores gêneros da tribo Clytini Mulsant, 1839 e, nas Américas, inclui 45 espécies largamente distribuídas do Canadá à Argentina (MonNÉ 1993; VIANA 1994; Di Iorio 1995, 1998). O gênero compreende dois subgêneros: M. (Megacyllene) Casey, 1912, com 40 espécies conhecidas e M. (Sierracyllene) Tippmann, 1960 com cinco espécies.

O material estudado pertence ao Museu Nacional, Universidade Federal do Rio de Janeiro, Rio de Janeiro (MNRJ).

\section{Megacyllene (Megacyllene) castroi (Prosen, 1947) comb. nov., stat. nov.}

(Fig. 1)

Cyllene Mellyi Castroi Prosen, 1947a: 255; 1947b: 326 (distr.); Monné, 1993: 8 (cat., in syn.).

Megacyllene (Megacyllene) cryptofrasciata Di Iorio, 1998: 5, figs. 1, 4. Syn. nov.

A comparação de um síntipo fêmea de Cyllene mellyi castroi Prosen, 1947 proveniente da Argentina, Santiago del Estero, Villa Unión, depositado no MNRJ via coleção Campos Seabra, com a descrição original e figura de $M$. cryptofrasciata Di Iorio, 1998, permitiu propor a presente sinonimia.

Megacyllene (M.) castroi (Fig. 1) difere de M. (M.) mellyi (Chevrolat, 1862) pela pubescência predominantemente castanho-amarelada; protórax densamente revestido de pilosidade amarela e, as fêmeas, com os lados do protórax regularmente arredondados, sem projeções látero-posteriores. $\operatorname{Em} M$. (M.) mellyi, predominam as pubescências de cor preta e vermelha, aveludadas e o protórax é revestido de pilosidade preta, esparsa e curta nos dois terços posteriores e cinzenta no terço anterior; além disso, os lados do protórax têm uma projeção romba no início do terço basal em ambos os sexos.
Di IoRIo $(1995: 323 ; 1998: 12)$ na chave de subgêneros e espécies de Megacyllene que ocorrem na Argentina não mencionou C. mellyi castroi.

Megacyllene (M.) castroi está distribuida nas províncias de Santiago del Estero (Prosen 1947b), Catamarca, Tucumán e Rio Negro, na Argentina (Di IoRIo 1998).

\section{Megacyllene (Sierracyllene) trifasciata Viana, 1994}

Megacyllene trifasciata Viana, 1994: 165, fig. 2.

Megacyllene (Sierracyllene) tafivallensis Di Iorio, 1998: 9, figs. 2, 3. Syn. nov.

A comparação entre as descrições originais, ilustrações e comentários fornecidos por VIANA (1994) e Di IoRIo (1998) não deixa dúvidas sobre a co-especificidade de $M$. trifasciata e $M$. (Sierracyllene) tafivallensis.

Di IoRIo $(1995,1998)$ não tratou de M. trifasciata Viana, 1994 em seus trabalhos sobre os Clytini argentinos e, ao descrever $M$. (S.) tafivallensis, mencionou, entre o material examinado, um parátipo fêmea de "Catamarca, Andalgalá, II1987, Viana leg.". Curiosamente, esse exemplar corresponde aos dados de localidade, data e coletor do alótipo de $M$. trifasciata depositado na coleção do Instituto de Investigaciones Entomológicas de Salta, Salta (INESALT) (VIANA 1994).

Espécie registrada para Catamarca (VIANA 1994), Tucumán e Jujuy (Di Iorio 1998), na Argentina.

\section{Megacyllene (Megacyllene) ebenina sp. nov.} (Figs. 2, 3)

Macho. Tegumento preto, revestido com pilosidade preta 

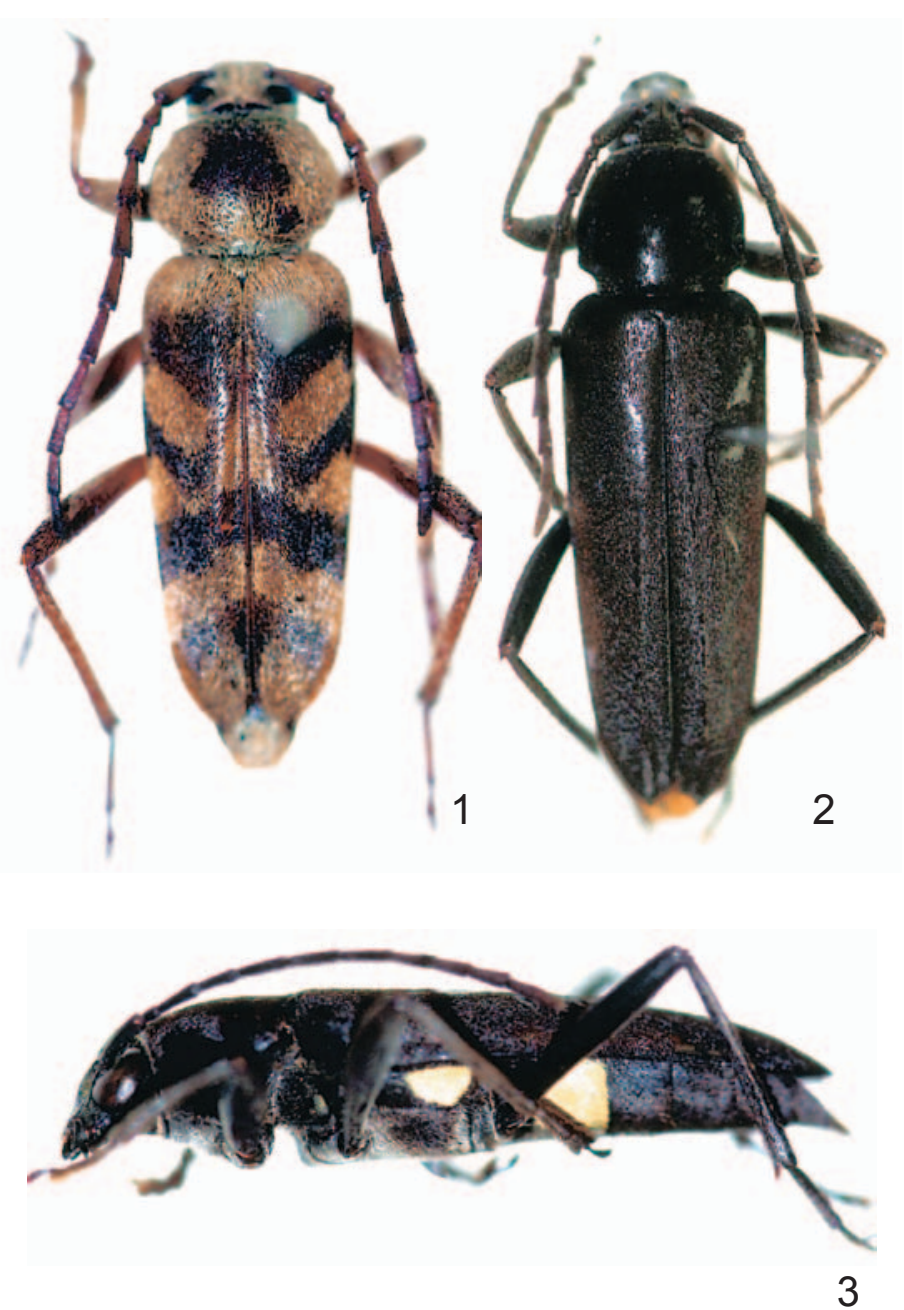

Figs. 1-3. 1, Megacyllene (Megacyllene) castroi (Prosen, 1947), síntipo fêmea; 2-3, M. (M.) ebenina sp. nov., holótipo macho, vistas dorsal e lateral (Steve Lingafelter foto).

no pronoto e acinzentada nos élitros e face ventral do corpo. Mancha de pubescência amarela recobre a metade posterior dos metepisternos e os lados do primeiro urosternito.

Antenas apenas ultrapassam o meio dos élitros; antenômeros II-V com curto espinho no lado interno da extremidade distal; II-VII com setas longas ápico-internas.
Lados do protórax subparalelos até o estreitamento no quarto posterior. Élitros com carena longitudinal paralela e próxima à sutura nos três quartos distais; ápices oblíqua e semicircularmente emarginados, ambos os ângulos com curto espinho. Processo prosternal truncado posteriormente; processo mesosternal em forte declive anterior. Meso- e metafêmures com dente curto a cada lado da extremidade distal.

Dimensões, em mm, macho: comprimento total 15,0; comprimento do protórax 3.0; maior largura do protórax 4,8; comprimento do élitro 10,0; largura umeral 5,2.

Holótipo macho, BRASIL, Santa Catarina: Corupá (Rio Vermelho), XII. 1956, A. Maller col. (MNRJ).

Comentários. M. (M.) ebenina sp. nov. separa-se de suas congêneres sul-americanas pelo tegumento preto e pubescência elitral acinzentada, sem vestígios de linhas ou máculas amarelas ou esbranquiçadas. M. (M.) falsa (Chevrolat, 1862) apresenta máculas de densa pubescência amarela na metade distal dos metepisternos e no primeiro urosternito, e o protórax e os élitros são ornados de linhas amarelas ou esbranquiçadas.

\section{REFERÊNCIAS}

Di Iorio, O. 1995. The Argentine species of the genus Megacyllene Casey, 1912 (Coleoptera, Cerambycidae), with description of a new species. Insecta Mundi 9(3-4): 317-328.

Di Iorio, O. 1998. New species, combinations, synonymies, and records of Clytini (Coleoptera: Cerambycidae). Insecta Mundi 12(1-2): $5-14$.

Monné, M. A. 1993. Catalogue of the Cerambycidae (Coleoptera) of the Western Hemisphere. Part IX. Subfamily Cerambycinae: Tribes Clytini, Anaglyptini, Tillomorphini and Cleomenini. São Paulo, Sociedade Brasileira de Entomologia, $131 \mathrm{p}$.

Prosen, A. F. 1947a. Nuevos cerambícidos argentinos (Col., Cerambycidae). Revista de la Sociedad Entomológica Argentina 13: 254-257.

Prosen, A. F. 1947b. Cerambycoidea de Santiago del Estero. Revista de la Sociedad Entomológica Argentina 13: 315-334.

VianA, M. J. 1994. Novedades sobre Clytini de la República Argentina (Coleoptera, Cerambycidae, Cerambycinae). Entomologia y Vectores 1(6): 163-166. 